\title{
Tunneling electron induced rotation of a copper phthalocyanine molecule on $\mathrm{Cu}(111)$
}

\author{
J. Schaffert, ${ }^{1}$ M. C. Cottin, ${ }^{1}$ A. Sonntag, ${ }^{1}$ C. A. Bobisch, ${ }^{1}$ R. Möller, ${ }^{1}$ J.-P. Gauyacq, ${ }^{2}$ and N. Lorente ${ }^{3,4}$ \\ ${ }^{1}$ Faculty of Physics, Center for Nanointegration Duisburg-Essen (CENIDE), University of Duisburg-Essen, \\ Lotharstr. 1, D-47057 Duisburg, Germany \\ ${ }^{2}$ Institut des Sciences Moléculaires d'Orsay, CNRS-Université Paris-Sud 11, UMR 8214, Bâtiment. 351, \\ Université Paris-Sud, F-91405 Orsay Cedex, France \\ ${ }^{3}$ ICN2-Institut Catala de Nanociencia i Nanotecnologia, Campus UAB, 08193 Bellaterra (Barcelona), Spain \\ ${ }^{4}$ CSIC-Consejo Superior de Investigaciones Cientificas, ICN2 Building, Campus UAB, 08193 Bellaterra (Barcelona), Spain
}

(Received 9 April 2013; revised manuscript received 17 June 2013; published 8 August 2013)

\begin{abstract}
The rates of a hindered molecular rotation induced by tunneling electrons are evaluated using scattering theory within the sudden approximation. Our approach explains the excitation of copper phthalocyanine molecules $(\mathrm{CuPc})$ on $\mathrm{Cu}(111)$ as revealed in a recent measurement of telegraph noise in a scanning tunneling microscopy experiment [Schaffert et al., Nat. Mater. 12, 223 (2013)]. A complete explanation of the experimental data is performed by computing the geometry of the adsorbed system, its electronic structure, and the energy transfer between tunneling electrons and the molecule's rotational degree of freedom. The results unambiguously show that tunneling electrons induce a frustrated rotation of the molecule. In addition, the theory determines the spatial distribution of the frustrated rotation excitation, confirming the striking dominance of two out of four molecular lobes in the observed excitation process. This lobe selectivity is attributed to the different hybridizations with the underlying substrate.
\end{abstract}

DOI: 10.1103/PhysRevB.88.075410

PACS number(s): 68.37.Ef, 72.10.-d, 79.20.Rf, 74.55.+v

\section{INTRODUCTION}

Tunneling electrons permit us to induce changes in atomic or molecular adsorbates at surfaces with great control. ${ }^{1-15}$ This breakthrough in fundamental science and in nanotechnology has stirred a lot of attention as it became possible to manipulate adsorbates and to induce reactions on the atomic scale. On the one hand, these tunneling experiments provide perfect toy systems to learn chemical rules; ${ }^{16,17}$ on the other hand, promising bottom-up techniques for creating nanometer-scaled devices become feasible. ${ }^{18}$

The first atomic manipulation experiments ${ }^{1,2}$ led to an important theoretical effort to unravel the mechanisms behind atomic motion induced by tunneling electrons. The task was complex due to the involvement of several length scales and the large number of degrees of freedom. Most treatments have favored master-equation approaches where the atomic and electronic degrees of freedom are perfectly separated and electronic transitions are incorporated only through atomic excitation and deexcitation rates. ${ }^{19-21}$ These rates are obtained through full quantum mechanical calculations, using electron-atom matrix elements fitted to or extracted from deformation-potential types of calculations in a golden-rule treatment. ${ }^{22}$ The atomic evolution is supposed to be that of a truncated harmonic potential, where the truncation indicates the rupture of an atomic bond. ${ }^{7,19}$ Only a few works have treated different atomic potential energy surfaces (PES) other than the truncated harmonic. Avouris et al. ${ }^{23}$ used atomic wave-packet propagations to evaluate the atomic dynamics in an excited PES after electron tunneling. More recently, the anharmonicity of the PES as revealed by density functional theory (DFT) was used to explain the electron induced motion of ammonia molecules on $\mathrm{Cu}(100){ }^{8,24}$ Despite the simplicity of these models, much insight was gained for single-atom and single-molecule dynamics.
Among the different evolutions of molecular dynamics on surfaces, rotations have proven to be complex. ${ }^{3,25}$ The origin of this difficulty can be traced back to the excitation mechanism itself. Instead of inducing a deformation of some localized bonds due to a brief charged state of the molecule, rotation implies some type of partial angular momentum transfer. Indeed, rotational excitation is closer to magnetic excitations than to vibrational excitations. ${ }^{15,25-27}$

By studying the telegraph noise observed in the tunneling current, Schaffert and co-workers ${ }^{15}$ have recently reported on the frustrated rotation of single copper phthalocyanine $(\mathrm{CuPc})$ molecules on $\mathrm{Cu}(111)$, excited by the tunneling current. Here, we present a theoretical account of this rotational excitation process. The adiabatic PES for the rotation of $\mathrm{CuPc}$ on $\mathrm{Cu}(111)$ is computed within DFT. We characterize the molecular adsorption as well as its electronic structure and calculate scanning tunneling microscope (STM) constant current images. These predictions are compared with the experimental observations ${ }^{15}$ and are used to explain them (Sec. II). The electron induced excitation is analyzed after the DFT structure calculations. A scattering theory account of the rotational excitation is presented. Using the customary approximation of the Tersoff-Hamann treatment of STM images, ${ }^{28}$ we obtain a DFT-based spatially resolved description of the rotational excitation by tunneling electrons (Sec. III). Here, we considerably expand the initial analysis of Ref. 15 by showing the interplay between the local hybridization of the relevant molecular orbitals with the substrate and the spatial dependence of the rotational excitation. We further complete the initial calculations and give a thorough exposition of the scattering theory, permitting us to have extra insight of the excitation process as well as the molecular dynamics induced by the tunneling electrons. The paper ends with a brief summary and concluding remarks. 


\section{STUDIED SYSTEM}

Our previous experimental and theoretical work ${ }^{15}$ shows that the tunneling current induces a frustrated rotational motion of $\mathrm{CuPc}$ molecules on $\mathrm{Cu}(111)$. An in-plane molecular axis changes by $\sim 7^{\circ}$ back and forth around the central $\mathrm{Cu}$ ion, which remains located at a highly symmetric bridge position on the surface. The rotation corresponds to transitions between the minimum energy conformation aligned with the $[-110]$ direction of the surface and two metastable local minima for the clockwise and anticlockwise rotations.

A brief summary on the experimental findings reads as follows. When $\mathrm{CuPc}$ (fourfold symmetric in the gas phase) is adsorbed on the sixfold-symmetric $\mathrm{Cu}(111)$ surface (just considering the topmost surface layer symmetry), the resulting STM images appear twofold symmetric. One pair of opposing benzopyrrole rings (lobes) appears pronounced as compared to the remaining two lobes. In the submonolayer regime, $\mathrm{CuPc}$ adsorbs individually, the formation of dimers or clusters is rather unlikely. In addition, the molecules appear partially fuzzy. The blurring of the molecular images is attributed to a molecular motion induced by the tunneling electrons. This is due to switching transitions between two discrete levels in the tunneling regime that correspond to two different tunneling currents for a fixed tip position. The switching events are random in time, with constant probabilities. ${ }^{15}$ Therefore, the definition of random telegraph noise (RTN) is fulfilled. The high-current level corresponds to nearly twice the low-current level. The switching frequency scales linearly with the tunneling current, hence, a one-electron driven process is observed.

A special electronic setup was designed to analyze the RTN signal from the tunneling current in real time, during the ongoing STM experiments. For a full characterization of a telegraph signal, the three quantities rate, amplitude, and duty cycle have to be measured. Details on this technique, scanning noise microscopy (SNM), are discussed in Ref. 29. The RTN characteristics can be obtained in spectroscopy measurements with fixed position of the tunneling tip, but they can also be mapped to create noise images exhibiting, e.g., spatially resolved excitation rates with the same resolution as the STM topography. The study of $\mathrm{CuPc}$ on $\mathrm{Cu}(111)$ yielded excitation maps clearly highlighting a lobe selectivity of the rotation excitation mechanism. Two out of the four molecular lobes appear noisy in STM. In SNM only those two lobes are visible with great detail. Based on the experimental results, the specific excitation of the two lobes could not be explained. However, the SNM amplitude maps as well as the spectroscopy of the duty cycle ${ }^{15}$ gave a strong hint for possible in-plane rotational motion by a small angle. That was the starting point for our DFT studies.

\section{DENSITY FUNCTIONAL THEORY DESCRIPTION}

In order to understand the induced rotation of $\mathrm{CuPc}$ on $\mathrm{Cu}$ (111), DFT studies were performed to obtain a maximum of information about the electronic and geometric properties of the molecule on the surface. The calculations were done using the VASP code. ${ }^{30}$

The calculations were performed for a $9 \times 10 \mathrm{Cu}$-atom unit cell with four layers. This large unit cell is required in order

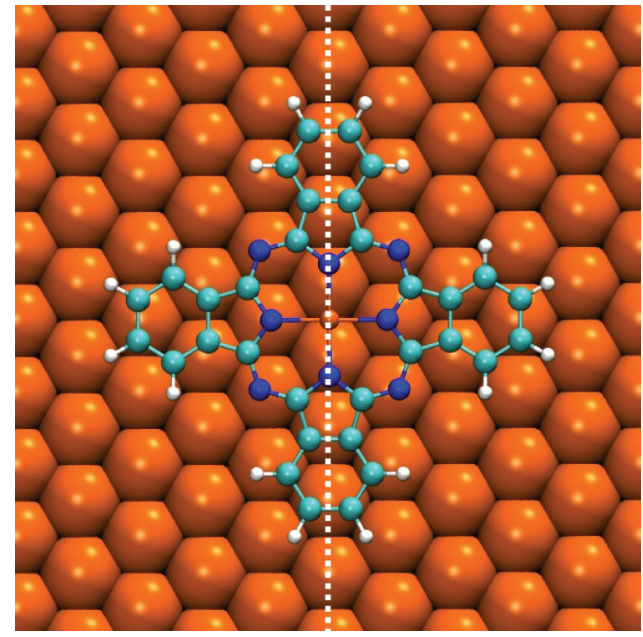

FIG. 1. (Color online) Minimum-energy conformation of a copper phthalocyanine $(\mathrm{CuPc})$ molecule adsorbed on $\mathrm{Cu}(111)$. The molecular $\mathrm{Cu}$ atom is located on a bridge site and two of the molecular lobes lie along the dense atomic row in $[-110]$ or equivalent direction (dashed white line along the $y$ axis of the figure) on the surface in agreement with the experimental findings (Ref. 15).

to reproduce dilute molecular densities. The large cell allows us to use a single $k$ point. The performed $\Gamma$-point calculations are equivalent to a $10 \times 9$ sampling of the one-atom surface cell. We have run a convergence test for the $\mathrm{Cu}$ (111) surface for the one-atom surface cell and found that the total energy was converged to better than $6 \mathrm{meV}$ with a $10 \times 9$ sampling. Hence, we expect the same accuracy for $\Gamma$-point calculations of the $9 \times 10 \mathrm{Cu}$-atom unit cell. Moreover, we will be mainly considering differences of energies in order to determine the rotational barrier, which will improve the convergence due to well-known error cancellations. The projector augmented wave (PAW) scheme for the atomic potentials ${ }^{31}$ was used, and the plane-wave basis set was expanded up to a cutoff energy of $300 \mathrm{eV}$.

We evaluated the total energy of different high-symmetry conformations of the adsorbed molecule. Among all of them the minimum energy corresponds to the molecular $\mathrm{Cu}$ atom sitting on a surface bridge site and one of the molecular symmetry axis aligned with the $[-110]$ direction (dense atomic row on the surface). Figure 1 shows the minimum energy configuration, in perfect agreement with the experimental observations. ${ }^{15}$ This adsorption conformation has also been found for $\mathrm{CoPc}$ on $\mathrm{Cu}(111)$ in a recent joint experimental and theoretical study ${ }^{32}$ as well as in other theoretical studies. ${ }^{33,34}$

\section{A. Adiabatic potential energy surface}

The PES as a function of the rotation angle $\phi$ between one of the molecular in-plane axes and the surface [-110] direction was computed by fixing one of the pyrrole-N atoms and the molecular $\mathrm{Cu}$ atom at their relaxed positions and rotating the corresponding interatomic axis to the desired angle. The structure was relaxed until all forces within the molecule and the first two substrate layers fell below $0.02 \mathrm{eV} / \AA$, keeping these two atoms fixed. Afterwards, the constrain on the pyrrole- $\mathrm{N}$ was released and placed on an aza-N atom, repeating the ionic convergence to the same thresholds. In this 


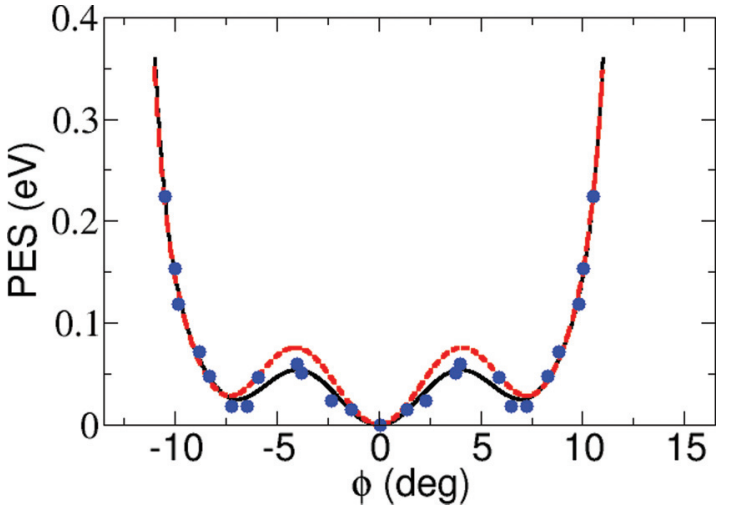

FIG. 2. (Color online) Adiabatic potential energy surface (PES) of a $\mathrm{CuPc}$ on $\mathrm{Cu}(111)$ as the molecule rotates around a surface normal defined by the position of its central $\mathrm{Cu}$ atom. Symbols correspond to computed values and lines are two different fits of the computed data. The two fits yield a frustrated rotation barrier between the equilibrium points at $\phi=0^{\circ}$ and $6.9^{\circ}$ of 50 and $70 \mathrm{meV}$, respectively.

way, the internal molecular and surface structures were relaxed for an extensive set of ionic relaxation calculations, excluding the back-relaxation to the original equilibrium state. Despite a careful convergence, we can not rule out a small $\left(\Delta \phi \approx \pm 0.1^{\circ}\right)$ uncertainty in the final positioning of the molecular axis. The PES shown in Fig. 2 reveals the existence of a metastable adsorption position of the $\mathrm{CuPc}$ rotated by $\pm 7^{\circ}$ from the equilibrium position. This secondary minimum is reminiscent of the geometry of full $\mathrm{CuPc}$ monolayers on $\mathrm{Cu}(111)$ where the molecule appears rotated by $\pm 7^{\circ}$ from the substrate axis. ${ }^{35,36}$

The calculation of the rotational barrier height is of particular importance for the determination of the rotational rates as shown below. Figure 2 shows the adiabatic PES calculated with the above prescriptions. In order to smoothen the small calculation uncertainties, a continuous function is fitted to the PES for later use in the dynamic calculations. Despite our efforts, we could fit different types of curves with different barriers. In the present calculations, we have used two different curves corresponding to rotational barriers of 50 and $70 \mathrm{meV}$ in order to study how critical the chosen PES is in the switching rate determination.

All of these calculations have been performed within the local density approximation (LDA). The rationale behind this is to use the overbinding error of LDA to obtain a physical molecule-surface distance, compensating to some extent the neglected van der Waals interaction in LDA. In order to evaluate the rotational excitation efficiency, it is highly desirable to describe the geometrical structure that corresponds to the underlying electronic structure, hopefully capturing the electronic molecule-substrate hybridization that drives the rotational excitation features. To this end, the self-consistent description of geometry and electronic structures as given by LDA permits us to link together geometry and electronic structures. This will prove to be important in the description of the excitation efficiencies as functions of the location of the injection point of the tunneling electron. However, it is well known that van der Waals interaction is a large component in the binding of large organic molecules such as phthalocyanines on noble-metal surfaces. ${ }^{37}$ In order to assess the accuracy
TABLE I. Computed height distances $z$ between the $\mathrm{Cu}$ central atom of the molecule and the surface plane and the molecular chemisorption energy $E_{\text {chem }}$ obtained with LDA and DFT-D2.

\begin{tabular}{llc}
\hline \hline & LDA & DFT+D2 \\
\hline$z(\AA)$ & 2.574 & 2.805 \\
$E_{\text {chem }}(\mathrm{eV})$ & 4.21 & 5.08 \\
\hline \hline
\end{tabular}

of the LDA PES, we repeated the calculations just for the $\phi=0^{\circ}$ case using DFT-D2 calculations including van der Waals interaction ${ }^{38}$ as implemented in the VASP code. Table I compares the results. The adsorption energy difference is $17 \%$, and the difference in adsorption distances is $8 \%$. As is well known, LDA compares favorably in these values with more realistic methods. And, particularly in the present case, the LDA PES should be a good estimation of the adiabatic PES because the missing van der Waals force is a long-range interaction which is little affected by atomic details such as a small-angle in-plane rotation of the molecular axis.

\section{B. Electronic structure}

Transition-metal phthalocyanines capture charge from noble-metal surfaces. ${ }^{37}$ Similarly, in the case of $\mathrm{CuPc}$, a full electron is captured into the first empty orbital of $\pi$ character ( $e_{2 g}$ in $D_{4 h}$ notation). This has clearly been seen for CuPc on $\mathrm{Ag}(100) .{ }^{37}$ Copper surfaces are more reactive than silver surfaces, hence more charge is captured. Our DFT results indicate a shift of the two $e_{2 g}$ orbitals below the Fermi energy, and the overall transferred charge approaches two electrons. Although the DFT Kohn-Sham orbitals strictly do not possess physical meaning, they are commonly used to evaluate the molecule's charge population. The results can be understood as a qualitative prediction that can be easily put to test, e.g., when computed constant current STM images are compared to the experiment. Indeed, as shown in Ref. 15, there is qualitative agreement between the Tersoff-Hamann simulated image ${ }^{28}$ and the experimental data. Here, we present calculations done within the Tersoff-Hamann theory but integrating over a given energy window:

$$
I \propto \sum_{\nu}\left|\psi_{\nu}(\vec{r})\right|^{2} F\left(E, \epsilon_{\nu}\right),
$$

where $E$ is the energy window from the Fermi energy $\left(E_{F}\right)$, $\psi_{v}(\vec{r})$ is an eigenstate of the full system's Hamiltonian with its eigenvalue $\epsilon_{\nu}$. The window function $F\left(E, \epsilon_{\nu}\right)$ is 1 if $\epsilon_{\nu}$ is between $E$ and $E_{F}$ and zero otherwise.

Figure 3 shows the evaluated constant current image using Eq. (1) for various values of $E$. For energy windows (equivalent to the applied STM bias) between -800 and $800 \mathrm{meV}$, the resulting images slightly vary. The images are dominated by the contribution of the extended $\pi$-like $e_{2 g}$ orbitals. Indeed, the density of states projected on these orbitals ${ }^{15}$ shows broad features spanning the above energy range. Due to a lack of wave-function amplitude on top of the molecular $\mathrm{Cu}$ atom, the STM image shows a depression at the molecular center. The characteristic four lobes resembling the two $e_{2 g}$ orbitals appear as protrusions. 

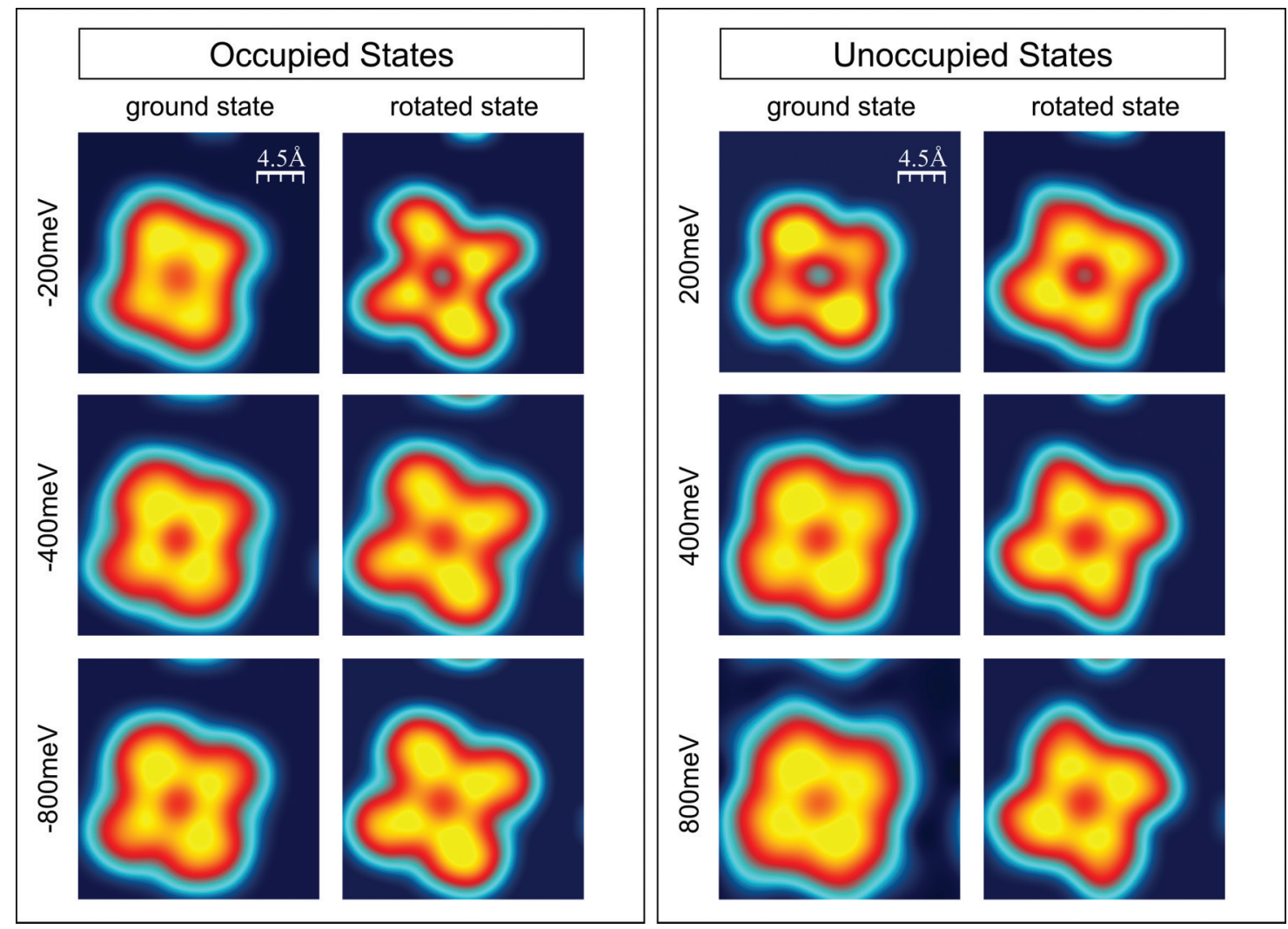

FIG. 3. (Color online) Constant current simulated images for different energy windows [Eq. (1)]. Both the molecule aligned with the $[-110]$ surface direction (now forming an angle of $30^{\circ}$ with the $y$ axis of the figure) and the molecule rotated by $\sim 7^{\circ}$ are shown. An image variation due to the different hybridization of the molecule with the substrate is apparent between the two types of molecules.

Figure 3 also showcases a comparison between the calculated images for the molecule aligned with the [-110] axis (the ground state or minimum energy configuration) and the molecule rotated by $\sim 7^{\circ}$ (metastable state). Only small variations can be seen. There are some slight asymmetries due to inaccuracies during the coordinate relaxation, but more significantly the rotated molecules show sharper images (smaller sized spots in the lobe region), indicative of a lower hybridization with the substrate. The simulated images also show a difference between the lobes aligned with the $[-110]$ axis and the perpendicular ones. The latter show a larger local density of states (see, for example, the ground-state image at $200 \mathrm{meV}$, and also at $-200 \mathrm{meV}$ ). This is due to the stronger interaction of the molecule with the surface along the $[-110]$ axis, and has connections with the difference between excitation probabilities as revealed in our excitation rate study below. Indeed, this finding relates to the experimentally observed factor of nearly 2, separating the high- and the low-current levels of the RTN tunneling current signal. The experimental factor of about 2 was reproduced by our DFT study when comparing cross sections within the calculated constant-height images.

\section{TUNNELING ELECTRON INDUCED ROTATION}

Energy transfer from a tunneling electron to an adsorbate has been studied in detail for various systems. ${ }^{17,39}$ In the case of vibrational excitation by electron collision, the resonant process associated with the transient capture of the electron by the target molecule has early been recognized to be very efficient for transferring energy from an electron to a molecule. Resonant processes are very active in electron collisions on free molecules (see, e.g., Ref. 40) or adsorbed molecules, ${ }^{41}$ as well as in tunneling conditions. ${ }^{42,43}$ For vibrational excitation, the large mass ratio between electron and atoms makes a recoil mechanism only weakly efficient and any trapping phenomenon (a resonance) which significantly increases the electron-molecule interaction time can boost the vibrational excitation efficiency. The situation is completely different in the case of rotational excitation. The rotational motion is slow, so that an electron-molecule interaction can be seen as a sudden process with the fast electron colliding with a fixed molecule. The excitation process is then related to a recoil phenomenon in which the scattered electron transfers recoil angular momentum to the target. The specificity of the rotational excitation compared to the vibrational excitation comes from the change in the relative orders of magnitude of electronic and nuclear momenta when going from linear to angular momenta.

In the case of a free molecule, quantization of the angular momentum forces the angular momentum of a scattered electron to be of the same order of magnitude as the expected value of the angular momentum of the low-energy rotational levels of the molecule, so that angular momentum exchange can be very efficient and lead to significant rotational excitation. ${ }^{44}$ In the present case, the nonspherical symmetry of 
the tunneling electrons and the frustrated rotation implies that neither the electron nor the molecule has a well-defined angular momentum: electron diffusion and molecular rotation are not associated with angular momentum eigenvectors. However, distributions of angular momentum can be associated with such systems, and the efficiency of the recoil in rotational excitation remains very high. ${ }^{25}$ One can stress that magnetic excitation by tunneling electrons also involves angular momentum transfer. Essentially, spin excitation and rotational excitation can be described along the same lines and both can be very efficient. ${ }^{26,27}$

\section{A. Scattering theory}

Let us consider the transition of an electron from an initial state $\left|\psi_{i}\right\rangle$ of energy $\epsilon_{i}$ in the STM tip into a final state in the sample $\left|\psi_{f}\right\rangle$ of energy $\epsilon_{f}$. The process may be inelastic such that $\epsilon_{i} \neq \epsilon_{f}$. The excess energy may then be transferred to a frustrated rotational state $\left|R_{n}\right\rangle$ of the molecule, defined as an eigenstate of the potential energy curve in Fig. 2. The molecule is excited from its frustrated rotation ground state $\left|R_{0}\right\rangle$ to an excited state $\left|R_{n}\right\rangle$, gaining an energy $E=E_{n}-E_{0}$ from the electron ( $E_{0}$ and $E_{n}$ are the energies of the initial and final rotational states). We can then say that the global system is initially in the state $|i\rangle=\left|R_{0}\right\rangle \otimes\left|\psi_{i}\right\rangle$ at energy $E_{i}=E_{0}+\epsilon_{i}$ and the final state and energy are $|f\rangle=\left|R_{n}\right\rangle \otimes\left|\psi_{f}\right\rangle$ and $E_{f}=$ $E_{n}+\epsilon_{f}$, respectively.

The transition rate per unit time for an inelastic electron transfer from tip to the substrate is given by the $T$ matrix

$$
\frac{1}{\tau_{\text {ine }}}=\frac{2 \pi}{\hbar} \sum_{i, f}\left|T_{i, f}\right|^{2} \delta\left(E_{i}-E_{f}\right) .
$$

In general, it is difficult to determine the connecting potential, and hence the $T$ matrix, in particular its inelastic part. However, in the present case, the molecular rotation is a slow motion compared to the electron transmission from the tip into the substrate. Therefore, one can use a sudden approximation in which the $T$ matrix is evaluated for fixed positions of the molecule with respect to the substrate (fixed $\phi$ angle) and then used to compute the $T$-matrix element between initial and final rotational states. This approach has been introduced very early in the treatment of rotational excitation of electron-free molecule collisions ${ }^{44}$ and later also used for adsorbed molecules. ${ }^{25}$ It is noteworthy that the $T$ matrix for a fixed position of the molecule with respect to the surface depends on all the molecular coordinates, and that this leads to a series of rotational and vibrational excitation processes. These could be treated with the present approach within the limits of the sudden approximation. Here, we single out the angular dependence and analyze the rotational excitation because the existence of the metastable adsorption geometry at $7^{\circ}$ from the equilibrium position permits us to observe it.

Expression (2) can be further simplified in the TersoffHamann approximation ${ }^{28}$ : the $T$ matrix for a fixed position of the molecule is taken proportional to the electronic wave function evaluated at the tip apex $\left(\vec{r}_{0}\right)$ :

$$
T_{i, f}=\left\langle\psi_{i}|\hat{T}| \psi_{f}\right\rangle \approx C \psi_{f}\left(\phi, \vec{r}_{0}\right),
$$

where $\psi_{f}\left(\phi, \vec{r}_{0}\right)$ is the molecule+substrate wave function. It is computed for an STM tip located at $\vec{r}_{0}$, a fixed position with respect to the substrate and for an angle $\phi$ of the molecule rotated from its equilibrium position. $C$ is the proportionality constant. We explicitly write the dependence of the wave function on the molecular angle $\phi$ (relative position of the molecule and substrate) for its later use in the evaluation of the rotational excitation.

We can now replace each quantity in expression (2) by its value:

$$
\begin{aligned}
\frac{1}{\tau_{\text {ine }}}= & \frac{2 \pi}{\hbar} \sum_{i, f} \sum_{n>N_{R}} f_{\text {tip }}\left(\epsilon_{i}\right)\left[1-f_{\text {sub }}\left(\epsilon_{f}\right)\right] \\
& \times\left|\left\langle R_{0}\left|\left\langle\psi_{i}|\hat{T}| \psi_{f}\right\rangle\right| R_{n}\right\rangle\right|^{2} \delta\left(E_{0}+\epsilon_{i}-E_{n}-\epsilon_{f}\right) .
\end{aligned}
$$

The rotational states $R_{n}(\phi)$ are evaluated by solving the Schrödinger equation in the adiabatic PES computed using DFT. In the summation, we explicitly defined electron flow from the tip to the substrate by using the Fermi factors of the tip $f_{\text {tip }}$ and the substrate $f_{\text {sub }}$. The index $N_{R}$ is that of the $R_{n}$ level above which the molecule actually rotates. Indeed, excitation from the ground state $R_{0}$ to low-lying rotational excited states $R_{n}$ is not sufficient to lift the molecule out of its equilibrium potential well (Fig. 2). The rotational excitation must be strong enough for the molecule to overcome the potential barrier separating the equilibrium well from the two metastable $\pm 7^{\circ}$ side wells (see Fig. 2). This limit corresponds to the level $N_{R}$ in the formulas. For the PES with a rotational barrier of $70 \mathrm{meV}$, $N_{R}$ is equal to 25 . This emphasizes that jumps from one well to the other have to involve highly excited states of the frustrated rotation and, thus, a very efficient rotational excitation process is needed.

Making use of (i) the Tersoff-Hamann approximation, (ii) Eq. (3), and (iii) the zero Kelvin expression for the Fermi distribution functions $\Theta\left(\epsilon_{F}-\epsilon\right)$, we can further simplify the expression by assuming that the tip is made of a material where the details of the band structure can be summarized in a density of states (DOS) function $D_{\text {tip }}(\epsilon)$. Finally, the rate for the excitation over the rotational barrier $1 / \tau_{\text {ine }}$ is

$$
\begin{aligned}
\frac{1}{\tau_{\text {ine }}}= & \frac{2 \pi}{\hbar} \sum_{n>N_{R}, f} D_{\text {tip }}\left(\epsilon_{f}+E\right)|C|^{2} \\
& \times\left|\left\langle R_{0}\left|\psi_{f}\left(\phi, \vec{r}_{0}\right)\right| R_{n}\right\rangle\right|^{2} \\
& \Theta\left(\epsilon_{F}+e V-\epsilon_{f}-E\right) \Theta\left(\epsilon_{f}-\epsilon_{F}\right) .
\end{aligned}
$$

The total electron current is equal to the electron transition rate from tip to substrate times the electron charge: $I=$ $e \times \frac{1}{\tau_{\mathrm{Tot}}}$, where $\frac{1}{\tau_{\mathrm{Tot}}}$ is similar to Eq. (5) except that the summation over the rotation index $n$ runs over the entire rotational spectrum. In the case of the total current, there is no restriction in the sum over $n$, while in the inelastic rate [Eq. (5)], the summation is restricted to the over-barrier levels $n>N_{R}$. We can define the fraction of the current that induces rotation from one potential well to another, also known as the inelastic electron fraction, by the ratio of the inelastic current to the total current:

$$
\eta\left(\vec{r}_{0}, V\right)=\frac{1}{\tau_{\text {ine }}} / \frac{1}{\tau_{\text {Tot }}} .
$$

Here, we assume that the constant $\mathrm{C}$ is the same for the inelastic and elastic channels following Ref. 43 where this approximation was shown to give a very good description of 
inelastic changes of conductance in electron induced vibrational excitations. Actually, the unicity of $\mathrm{C}$ is just a consequence of the use of the sudden approximation. Equation (3) defines the $T$ matrix introducing the factor $\mathrm{C}$ and this equation is later used to evaluate elastic and inelastic components of the tunneling current, so that the $C$ factor is the same for the two components. The benefit from the implementation of the inelastic fraction is that the unknown constant $C$ in Eq. (3) and the tip's DOS factor cancel out under the assumption of a roughly constant DOS of the tip over the energy range of interest.

Using the above expressions, the inelastic electron fraction becomes

$$
\eta\left(\vec{r}_{0}, V\right)=\frac{\sum_{n>N_{R}, f}\left|\left\langle R_{0}\left|\psi_{f}\left(\phi, \vec{r}_{0}\right)\right| R_{n}\right\rangle\right|^{2} \Theta\left(\epsilon_{F}+e V-\epsilon_{f}-E\right) \Theta\left(\epsilon_{f}-\epsilon_{F}\right)}{\sum_{n, f}\left|\left\langle R_{0}\left|\psi_{f}\left(\phi, \vec{r}_{0}\right)\right| R_{n}\right\rangle\right|^{2} \Theta\left(\epsilon_{F}+e V-\epsilon_{f}-E\right) \Theta\left(\epsilon_{f}-\epsilon_{F}\right)} .
$$

The inelastic electron fraction $\eta$ does not evaluate the number of molecules that actually do rotate, but rather the fraction of electrons that excite the molecule over a certain rotational level $N_{R}$. Indeed, on the time scale of the experiment, a molecule excited by a tunneling electron from the rotational ground state $R_{0}$ to a rotational level $R_{n}$ will quickly relax to a lower state, i.e., it will end up localized in one of the three potential wells and the experimental transition rate concerns transitions between these potential wells. Relaxation from level $R_{n}$ can involve collisions with substrate electrons (electron-hole pair creation in a process very similar to the one discussed here) or the transfer of the rotational excitation (coordinate $\phi$ ) to other degrees of freedom of the heavy particles in the molecule-substrate system (intramolecular relaxation or transfer to phonons). Figure 4 shows a simplified scheme of the excitation/deexcitation process leading to the transfer from the equilibrium potential well to another. For simplicity, only one intermediate level in the relaxation is displayed in the figure.

We did not try to evaluate in detail this very complex relaxation process but resort to a geometrical statistical approximation. We evaluate the branching ratio for the deexcitation from the level $R_{n}$ towards a given metastable well in terms of

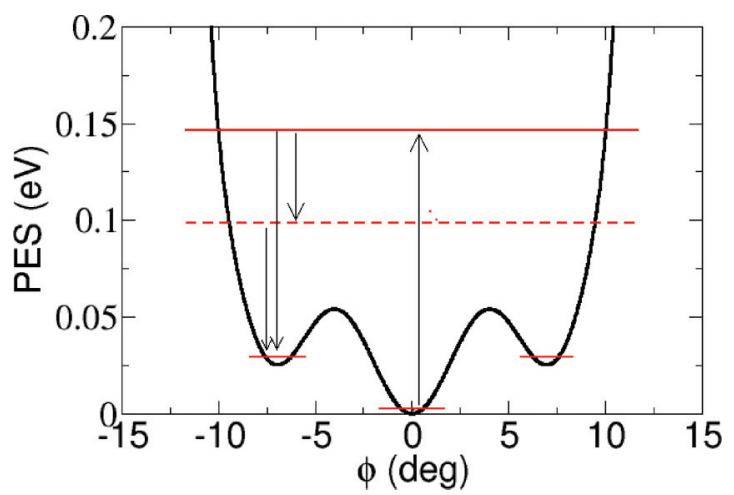

FIG. 4. (Color online) General excitation and deexcitation scheme in-between rotational levels within the potential energy surface leading to the molecular frustrated rotation. A direct deexcitation from the highly excited level (upper red solid line) to the left side well is indicated by an arrow. The deexcitation can also take place via many intermediate states depending on the degree of excitation. Here, we choose one intermediate state (dotted line) to exemplify the mediated two-step deexcitation. the weight of the rotational wave function $R_{n}$ in the $\phi$ range of the metastable well, approximated as the $\phi$ region beyond a critical angle $\phi_{c}$. By inspection of the PES, we choose $\phi_{c}=5^{\circ}$. The fraction of molecules excited to a given level $n$ that are eventually trapped in the right well is

$$
p_{R}(n)=\int_{\phi_{c}}^{15^{\circ}}\left|R_{n}(\phi)\right|^{2} d \phi / \int_{-15^{\circ}}^{15^{\circ}}\left|R_{n}(\phi)\right|^{2} d \phi,
$$

and we can assume that the same probability rules the trapping in the left well. The $\pm 15^{\circ}$ limits reflect that the molecule is not freely rotating above the surface, but it is confined to a $30^{\circ}$ sector due to the molecule+substrate joint symmetry and to the high barrier separating the equivalent $\phi$ regions (see Fig. 2).

Figure 5 shows the probability of deexcitation into the right well from the level $n$ of the rotational PES, $p_{R}(n)$. For low $n$, the excited states are localized in the equilibrium well so that the branching ratio is equal to zero. Above the energy of the bottom of the metastable wells, some of the states are localized in the side wells. Owing to our approach which considers even and odd functions, the branching ratio is equal to 0.5 for these states. Above the frustrated rotation barrier $(70 \mathrm{meV}$ for the shown case), i.e., for the states included in the summation in Eq. (7), the probability steadily increases to $\frac{1}{3}$, consistent with a roughly equal partitioning among the wells.

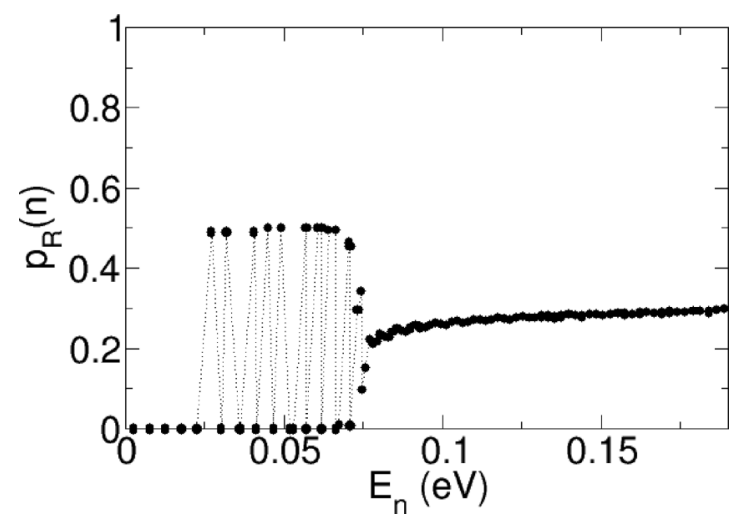

FIG. 5. Probability of deexcitation into the right well from the level $n$ of the rotational PES [Eq. (8)]. For levels below the 70-meV barrier there is an alternation of levels among left and right and central wells. Above the barrier, the rotational states become increasingly delocalized about all wells and the probability to find the molecule in the right well approaches $\frac{1}{3}$. 
The inelastic fraction of electrons that make the molecule jump from the equilibrium well into the right well $\eta_{R}$ is then obtained by including the branching ratio $p_{R}(n)$ into the expression (7):

$$
\eta_{R}\left(\vec{r}_{0}, V\right)=\frac{\sum_{n>N_{R}, f} p_{R}(n)\left|\left\langle R_{0}\left|\psi_{f}\left(\phi, \vec{r}_{0}\right)\right| R_{n}\right\rangle\right|^{2} \Theta\left(\epsilon_{F}+e V-\epsilon_{f}-E\right) \Theta\left(\epsilon_{f}-\epsilon_{F}\right)}{\sum_{n, f}\left|\left\langle R_{0}\left|\psi_{f}\left(\phi, \vec{r}_{0}\right)\right| R_{n}\right\rangle\right|^{2} \Theta\left(\epsilon_{F}+e V-\epsilon_{f}-E\right) \Theta\left(\epsilon_{f}-\epsilon_{F}\right)} .
$$

At this point, one can stress that the quantity $\eta_{R}$ can be directly compared with the equivalent quantity in the experiment (Ref. 15). Furthermore, the excitation probability $\eta_{R}\left(\vec{r}_{0}, V\right)$ depends on where the tip apex is located, $\vec{r}_{0}$, i.e., expression (9) provides a geometrical map of the excitation process.

As was experimentally shown, ${ }^{15}$ the derivative of the efficiency with respect to the bias gives density of states information. This can easily be comprehended, as the derivative changes one $\Theta$ function in expression (9) into a delta function. The obtained quantity strongly resembles the local density of states with information on the involved rotational states.

The above treatment parallels that used to compute the changes in conductance due to magnetic excitations (see a review in Ref. 27). Indeed, both cases deal with an angular momentum transfer: in the present case, the rotational angular momentum of an adsorbed molecule, and in the magnetic case the spin of a magnetic molecule. The theoretical treatments just evaluate the sharing of the electron flux among the different rotational or magnetic channels, respectively.

\section{B. Results}

Figure 6 shows the spatial distribution of the inelastic fraction of electrons exciting the molecular rotation. The maximum corresponds to $\sim 2.5 \times 10^{-5}$ for a bias of $-0.5 \mathrm{~V}$. The equivalent experimental number ${ }^{15}$ is rather $\sim 1.2 \times 10^{-7}$ at $-0.6 \mathrm{~V}$. The calculations have been performed for $\mathrm{a}$

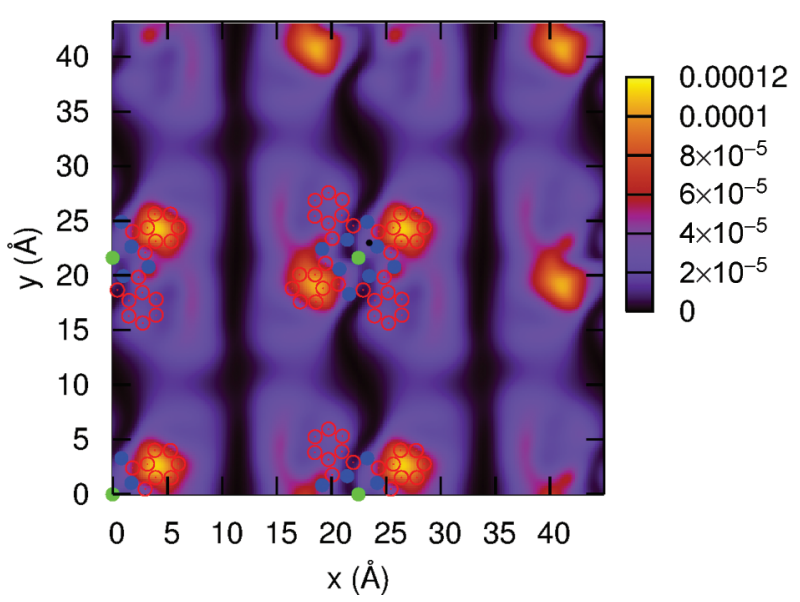

FIG. 6. (Color online) Inelastic fraction of electrons inducing the molecular rotation. The contributions from all the excited rotational states to the transition from the equilibrium well to one of the lateral metastable wells are summed, using Eq. (9). The figure contains two calculation supercells and the atomic axis $[-110]$ on the surface is rotated by $30^{\circ}$ with respect to the $y$ axis of the figure. rotational barrier of $70 \mathrm{meV}$. As we show in Fig. 2, there is some uncertainty in the determination of the rotational barrier and $50 \mathrm{meV}$ is also consistent with our computed PES. As expected, a change in the barrier height strongly influences the rate. Indeed, the rotational rate for a tip located at the maximum efficiency spot changes by a factor of $\sim 3$ between the 70 - and the 50-meV barriers. This is probably one of the largest sources of uncertainty in the present calculation. Another uncertainty is hidden inside the PES calculations of Sec. II A. It corresponds to the PES as a function of the angle $\phi$ when all the other coordinates have been relaxed, so that it includes an implicit adiabatic assumption for these coordinates, justified by the slowness of the rotational motion. In addition, in the presence of distortions of the molecular geometry as the angle $\phi$ is varied, a more complex Schrödinger equation should be used than the pure rotation one used above to define the $R_{n}$ wave functions. In the present case, the distortions of the molecular frame are below $1 \mathrm{pm}$ during the rotation, hence justifying the use of a single variable in the Schrödinger equation.

The calculation involves the integration over angle $\phi$, the PES rotational levels, and the electronic wave function $\psi_{f}\left(\phi, \vec{r}_{0}\right)$. Since each angular value of $\psi_{f}\left(\phi, \vec{r}_{0}\right)$ involves a full self-consistent calculation of the molecule on the surface for that angle, it is a very costly calculation to perform. However, high- $R_{n}$ rotational states exhibit a large number of nodes and thus require a large number of $\phi$ values for the integrals in Eq. (9). We balanced accuracy and computation costs by discretizing the integration with nine $\phi$ values at which full DFT calculations were performed. We then used a linear interpolation of $\psi_{f}$ between the ab initio points. In this way, we can have a double scale of integration points for the $\psi_{f}$ and $R_{n}$ parts of the integral.

The calculation shows that the two molecular lobes aligned perpendicular to the dense atomic row direction $[-110]$ yield the largest rotational rate, in excellent agreement with the experiment. ${ }^{15}$ The ratio of rates between the lobes perpendicular to [-110] and the parallel ones is roughly 5 (Fig. 6), in very good agreement with the experimental one. By studying the electronic states of molecule and surface and their relaxed geometry, we conclude that it is the local binding of the molecule to the surface that determines the efficiency of the angular momentum transfer, i.e., the excitation mechanism is a local electronic effect. This is illustrated in Fig. 7.

Figure 7 shows the main features of the hybridized molecule-substrate electronic structure. The PDOS onto the $\mathrm{e}_{2 g}$ orbital in alignment with the [-110] direction is depicted in (a) and the second $e_{2 g}$ orbital, which is aligned with the perpendicular direction is plotted in (d). A broader PDOS structure is found in Fig. 7(a). This is the signature of a stronger hybridization of the $e_{2 g}$ orbital along the perpendicular lobe 

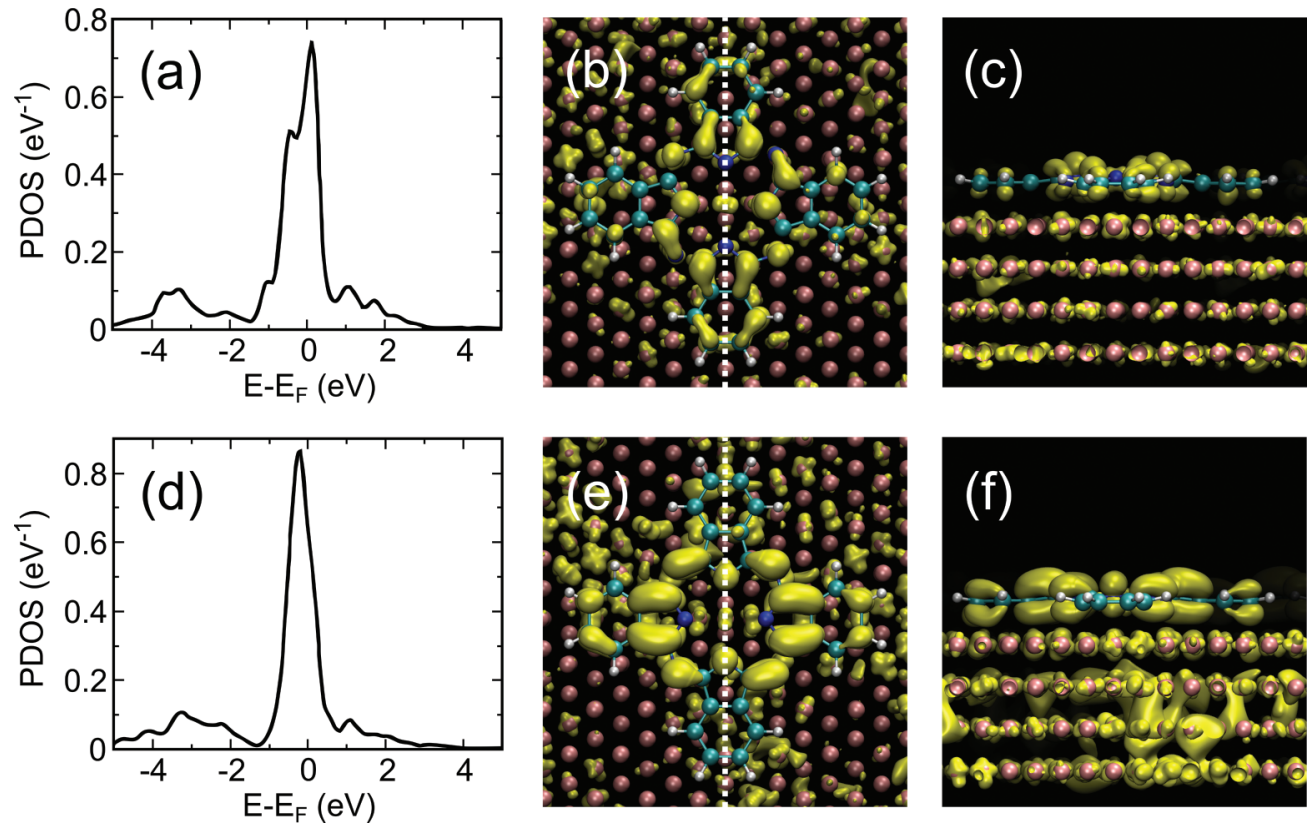

FIG. 7. (Color online) Electronic structure giving rise to the lobe selectivity in the electron excitation. (a) Projected density of states (PDOS) on the free molecule lowest unoccupied molecular orbital (LUMO), the $e_{2 g}$ orbital aligned with the [ -110 ] direction ( $y$ axis of the figure). (b) Top view of the modulus square of the electronic eigenfunction corresponding to the distorted LUMO with an energy close to the maximum of the PDOS. (c) Side view of the same wave function from the [-110] direction. (d) PDOS on the free molecule $e_{2 g}$ orbital aligned perpendicular to the $[-110]$ direction. (e) Top view of the corresponding electronic eigenfunction and $(f)$ side view from the $[-110]$ direction.

with the $\mathrm{Cu}$ substrate. This is corroborated by the plots of the modulus square of the wave functions for the orbitals close to Fermi level and exhibiting a large overlap with the $e_{2 g}$ of the free molecule. Indeed, the splitting of the two $e_{2 g}$ orbitals due to their interaction with the substrate significantly alters the shape of the orbitals. Despite this distortion, it appears that the image Fig. 7(c) displays little angle-dependent hybridization between the $e_{2 g}$ orbital and the substrate, while Fig. 7(f) shows a sizable angle-dependent hybridization at the same plotted surface isovalue. This stronger angular dependence of the $e_{2 g}$-substrate hybridization leads to a more pronounced $\phi$ dependence of $\psi_{f}\left(\phi, \vec{r}_{0}\right)$. Indeed, rotating the molecule will strongly perturb the bonds originating from the $e_{2 g}$ orbital perpendicular to the [-110] direction. Hence, the response to an injected electron is stronger for this last orbital. The enhanced dependence of the dynamical response with the active coordinate has been shown to be also at the origin of the stronger inelastic electron tunneling (IET) signals in vibrational inelastic electron tunneling spectroscopy (IETS). ${ }^{45}$

\section{CONCLUSIONS}

The DFT study on $\mathrm{CuPc}$ on $\mathrm{Cu}(111)$ yields a molecular adsorption conformation in good agreement with the experimental data. ${ }^{15}$ The molecule is adsorbed flat on the surface with the central $\mathrm{Cu}$ atom sitting on a highly symmetric bridge site with two out of the four molecular lobes aligned along the densely packed [-110], [0-11], or [10-1] directions. No buckled or bent conformation is found. In addition, we calculated the evolution of the total energy of the system when the molecule is rotated around a surface normal, going through the central atom of the molecule. The resulting PES reveals the existence of two local minima for symmetrical rotations by $\pm 7^{\circ}$ clockwise and anticlockwise from the equilibrium position. The molecule can undergo a frustrated rotational motion around its equilibrium position as soon as it gains an energy higher than the rotational barrier $(70 \mathrm{meV})$ by flipping from the equilibrium well into one of the side wells of the PES as depicted in Fig. 2. These computational results confirm the experimental interpretation that the blurred STM images of one opposite pair of benzopyrrole rings of $\mathrm{CuPc}$ are caused by jumps of the molecule between three adsorption wells induced by tunneling electrons. ${ }^{15}$

The simulated STM images show that the four $\mathrm{CuPc}$ lobes are not equivalent but split into two groups (lobes along the dense atomic rows and perpendicular to it). Their position relative to the underlying $\mathrm{Cu}$ lattice is different and consequently they hybridize differently with the substrate. The strong hybridization of the $\pi$-like orbitals located on the molecular lobes leads to a sizable charge transfer of two electrons from the surface to the molecule. As a result, the simulated STM image is dominated by the contribution of the $e_{2 g}(\pi$-like) orbitals of the molecule. This contribution is slightly different on the two types of lobes in good agreement with the symmetry reduction observed in the experiment.

By analyzing the telegraph noise in the tunneling current, which causes a fuzzy appearance of CuPc in STM images, Ref. 15 showed that the molecular rotation rate is maximal on the two lobes perpendicular to the dense atomic row $[-110] \mathrm{di}$ rection, whereas rotation can not effectively be induced on the remaining lobes. We have performed calculations that revealed the excitation mechanism of tunneling electrons inducing the 
frustrated rotational dynamics and leading to fluctuations in the STM tunneling current signals. Our approach is based on the sudden approximation for the rotation and the Tersoff-Hamann approximation for the $T$ matrix in the tunneling regime. The main result of the theory is that the tunneling electron has a finite probability of exciting the molecular rotation: the electron is briefly in contact with the molecule and departs leaving it in one of the rotational levels; when the excited rotational level is above the potential energy barrier separating the three frustrated rotation wells, then the jump of the molecule from one well to another becomes possible. This process is analogous to the one found in magnetic excitation by tunneling electrons, where the adsorbate spin changes depending on the weight of each magnetic state in the tunneling symmetry. ${ }^{26,27}$ Our calculations show a clear difference in the excitation rates over the two types of molecular lobes, identical to the selectivity observed experimentally. We connect this selectivity with the different hybridization between the $e_{2 g}$ orbitals (mainly located on the molecular lobes) and the substrate. These different hybridizations lead to different rotational rates. The lobe selectivity observed in the telegraph noise induced by tunneling electrons thus appears to be of electronic origin.

\section{ACKNOWLEDGMENTS}

J.S., A.S., C.A.B., and R.M. gratefully acknowledge financial support by the Deutsche Forschungsgemeinschaft through the SFB616 'Energy Dissipation at Surfaces.' N.L. is supported by the ICT-FET Integrated Project AtMol (http://www.atmol.eu). M.C.C. thanks the Studienstiftung des deutschen Volkes.
${ }^{1}$ D. M. Eigler, C. P. Lutz, and W. E. Rudge, Nature (London) 352, 600 (1991).

${ }^{2}$ G. Dujardin, R. E. Walkup, and P. Avouris, Science 255, 1232 (1992)

${ }^{3}$ B. C. Stipe, M. A. Rezaei, and W. Ho, Science 279, 1907 (1998).

${ }^{4}$ J. K. Gimzewski, C. Joachim, R. R. Schlittler, V. Langlais, H. Tang, and I. Johannsen, Science 281, 531 (1998).

${ }^{5}$ S.-W. Hla, L. Bartels, G. Meyer, and K.-H. Rieder, Phys. Rev. Lett. 85, 2777 (2000).

${ }^{6}$ L. Bartels, M. Wolf, T. Klamroth, P. Saalfrank, A. Kühnle, G. Meyer, and K.-H. Rieder, Chem. Phys. Lett. 313, 544 (1999).

${ }^{7}$ T. Komeda, Y. Kim, M. Kawai, B. N. J. Persson, and H. Ueba, Science 295, 2055 (2002).

${ }^{8}$ J. I. Pascual, N. Lorente, Z. Song, H. Conrad, and H.-P. Rust, Nature (London) 423, 525 (2003).

${ }^{9}$ J. A. Stroscio and R. J. Celotta, Science 306, 242 (2004).

${ }^{10}$ Y. Sainoo, Y. Kim, T. Okawa, T. Komeda, H. Shigekawa, and M. Kawai, Phys. Rev. Lett. 95, 246102 (2005).

${ }^{11}$ V. Iancu and S.-W. Hla, Proc. Natl. Acad. Sci. USA 103, 13718 (2006).

${ }^{12}$ L. Gao, Q. Liu, Y. Y. Zhang, N. Jiang, H. G. Zhang, Z. H. Cheng, W. F. Qiu, S. X. Du, Y. Q. Liu, W. A. Hofer et al., Phys. Rev. Lett. 101, 197209 (2008).

${ }^{13}$ K. Motobayashi, Y. Kim, H. Ueba, and M. Kawai, Phys. Rev. Lett. 105, 076101 (2010).

${ }^{14}$ T. Kumagai, A. Shiotari, H. Okuyama, S. Hatta, T. Aruga, I. Hamada, T. Frederiksen, and H. Ueba, Nat. Mater. 11, 167 (2012).

${ }^{15}$ J. Schaffert, M. C. Cottin, A. Sonntag, H. Karacuban, C. A. Bobisch, N. Lorente, J.-P. Gauyacq, and R. Möller, Nat. Mater. 12, 223 (2013).

${ }^{16}$ H. J. Lee and W. Ho, Science 286, 1719 (1999).

${ }^{17}$ W. Ho, J. Chem. Phys. 117, 1033 (2002).

${ }^{18}$ C. Joachim, J. K. Gimzewski, and A. Aviram, Nature (London) 408 , 541 (2000).

${ }^{19}$ S. Gao, M. Persson, and B. I. Lundqvist, Solid State Commun. 84, 271 (1992).

${ }^{20}$ R. Walkup, D. Newns, and P. Avouris, J. Electron Spectrosc. Relat. Phenom. 6465, 523 (1993).

${ }^{21}$ G. P. Salam, M. Persson, and R. E. Palmer, Phys. Rev. B 49, 10655 (1994).
${ }^{22}$ S. Gao, M. Persson, and B. I. Lundqvist, Phys. Rev. B 55, 4825 (1997).

${ }^{23}$ P. Avouris, R. Walkup, A. Rossi, T.-C. Shen, G. Abeln, J. Tucker, and J. Lyding, Chem. Phys. Lett. 257, 148 (1996).

${ }^{24}$ N. Lorente and J. I. Pascual, Philos. Trans. R. Soc., Ser. A 362, 1227 (2003).

${ }^{25}$ D. Teillet-Billy, J. P. Gauyacq, and M. Persson, Phys. Rev. B 62, R13306 (2000).

${ }^{26}$ N. Lorente and J.-P. Gauyacq, Phys. Rev. Lett. 103, 176601 (2009).

${ }^{27}$ J. P. Gauyacq, N. Lorente, and F. D. Novaes, Prog. Surf. Sci. 87, 63 (2012).

${ }^{28}$ J. Tersoff and D. R. Hamann, Phys. Rev. Lett. 50, 1998 (1983)

${ }^{29}$ J. Schaffert, M. C. Cottin, A. Sonntag, H. Karacuban, D. Utzat, C. A. Bobisch, and R. Möller, Rev. Sci. Instrum. 84, 043702 (2013).

${ }^{30}$ G. Kresse and J. Furthmüller, Phys. Rev. B 54, 11169 (1996).

${ }^{31}$ G. Kresse and D. Joubert, Phys. Rev. B 59, 1758 (1999).

${ }^{32}$ B. W. Heinrich, C. Iacovita, T. Brumme, D.-J. Choi, L. Limot, M. V. Rastei, W. A. Hofer, J. Kortus, and J.-P. Bucher, J. Phys. Chem. Lett. 1, 1517 (2010)

${ }^{33}$ X. Chen and M.Alouani, Phys. Rev. B 82, 094443 (2010).

${ }^{34}$ J. Z. Li, B.Li and J. G. Huo, Acc. Chem. Res. 43, 954 (2010),

${ }^{35}$ D. G. de Oteyza, A. El-Sayed, J. M. Garcia-Lastra, E. Goiri, T. N. Krauss, A. Turak, E. Barrena, H. Dosch, J. Zegenhagen, A. Rubio et al., J. Chem. Phys. 133, 214703 (2010).

${ }^{36}$ J. C. Buchholz and G. A. Somorjai, J. Chem. Phys. 66, 573 (1977).

${ }^{37}$ A. Mugarza, R. Robles, C. Krull, R. Korytár, N. Lorente, and P. Gambardella, Phys. Rev. B 85, 155437 (2012).

${ }^{38}$ S. Grimme, J. Comput. Chem. 27, 1787 (2006).

${ }^{39}$ T. Komeda, Prog. Surf. Sci. 78, 41 (2005).

${ }^{40}$ G. J. Schulz, Rev. Mod. Phys. 45, 423 (1973).

${ }^{41}$ V. Djamo, D. Teillet-Billy, and J. P. Gauyacq, Phys. Rev. Lett. 71, 3267 (1993).

${ }^{42}$ B. N. J. Persson and A. Baratoff, Phys. Rev. Lett. 59, 339 (1987).

${ }^{43}$ N. Lorente and M. Persson, Phys. Rev. Lett. 85, 2997 (2000).

${ }^{44}$ R. A. Abram and A. Herzenberg, Chem. Phys. Lett. 3, 187 (1969).

${ }^{45}$ N. Lorente and M. Persson, Faraday Discuss. 117, 277 (2000). 